\title{
A CLOSED ORBIT MEASUREMENT WITH THE NSRL BPM SYSTEM*
}

\author{
J. H. Wang", Y. Yin ${ }^{+}$, J. Y. Li, Z. P. Liu, B. G. Sun, G. C. Wang, J. H. Liu \\ NSRL, USTC, Hefei, Anhui 230029, P.R.China
}

\begin{abstract}
The beam position monitor system (BPM) of NSRL electron storage ring have a resolution of 10 microns with an accuracy of 50 microns, which is adequate for beam position measurement and the closed orbit correction. The paper describes both of the BPM system and the closed orbit measurement.
\end{abstract}

\section{INTRODUCTION}

The electron storage ring of NSRL of the University of Science and Technology of China at Hefei, P.R.China is a special synchrotron radiation facility. It operates with the energy of $800 \mathrm{MeV}$ and the beam current of 100$300 \mathrm{~mA}$. As known to all that the BPM system is one of key measuring equipment for advancing the quality of beam current and insuring the machine in normal operation. And the BPM system of NSRL has constructed early and its electronics have been modified now. The signals to noise ratio and the dynamic range of the measurement have been greatly increase after the modified electronics. The accuracy and the reliability of the system have been improved, which have the accuracy of $50 \mu \mathrm{m}$ and a resolution of $10 \mu \mathrm{m}$. With the help of the system we have finished the commission of insertion devices of NSRL storage ring [1]. The experiments prove that is adequate measurement of beam position and closed-orbit correction [2].

\section{THE BPM SYSTEM AND ITS SIGNAL PROCESSING}

\subsection{The BPM system and Calibration}

The circumference of the NSRL electron storage ring is 66 meter. The ring contains 12 dipole magnets and 48 quadrupole magnets, with 27 BPM. Each BPM has four button type pick-ups mounted in a skew manner as shown in Figure 1. The diameter of the button is $25 \mathrm{~mm}$. Each BPM button is welded to a BNC vacuum feedthrough, which then is welded to beam pipe. The beam pipes are 1.8 meter long with BPMs on both ends. The calibration has been done for each BPM. A wire

*Work supported by China Academy of Science contract KJ85

" Email: wjhua@ustc.edu.cn

${ }^{+}$Present address: Y.Y.Labs.Inc, P.O.Box 597, Fremont, CA 945370597,USA goes through the centre of the pipe to simulate the beam.

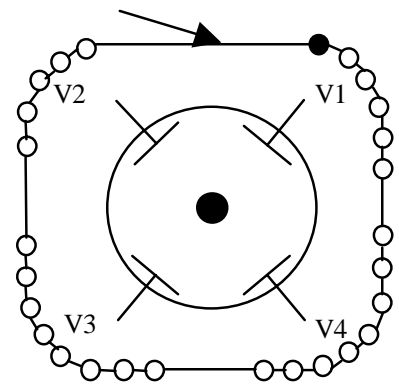

Figure 1: Distribution of BPM along ring and Single construction of BPM

The wire can be moved inside the beam pipe. A homemade electronics circuit detect the induce voltage from each pick-up for each position of the wire. In the way, two BPMs at the both ends of beam pipe can be Calibrated simultaneously. A computer recorded the voltage readings, and calculates the offset between the electronic and mechanical centre.

For the 25 mapped data, a least square method is used to calculate the coefficients $A_{n}$. The calculation is shown below [3]:

$U=\left(v_{2}-v_{1}+v_{3}-v_{4}\right) /\left(v_{1}+v_{2}+v_{3}+v_{4}\right)$

$V=\left(v_{1}-v_{4}+v_{2}-v_{3}\right) /\left(v_{1}+v_{2}+v_{3}+v_{4}\right)$

$X_{I}=\sum_{i=0}^{N} \sum_{j=0}^{i} a_{i-j, j}\left(U_{I}-U_{0}\right)^{i-j}\left(V_{I}-V_{0}\right)^{j}, U_{0}=\left.U_{I}\right|_{x=0}, I=1, \ldots, 25$

$Y_{I}=\sum_{i=0}^{N} \sum_{j=0}^{i} b_{i-j, j}\left(U_{I}-U_{0}\right)^{i-j}\left(V_{I}-V_{0}\right)^{j}, V_{0}=\left.V_{I}\right|_{y=0}$,

make $N=3$

$X$ or $Y=A_{1}+A_{2}\left(U_{I}-U_{0}\right)+A_{3}\left(V_{I}-V_{0}\right)+A_{4}\left(U_{I}-U_{0}\right)^{2}$

$$
\begin{aligned}
& +A_{5}\left(V_{I}-V_{0}\right)^{2}+A_{6}\left(V_{I}-V_{0}\right)\left(U_{I}-U_{0}\right)+A_{7}\left(U_{I}-U_{0}\right)^{3} \\
& +A_{8}\left(U_{I}-U_{0}\right)^{2}\left(V_{I}-V_{0}\right)+A_{9}\left(U_{I}-U_{0}\right)\left(V_{I}-V_{0}\right)^{2}+A_{0}\left(V_{I}-V_{0}\right)^{3}
\end{aligned}
$$

\subsection{The performance of the signal processing electronics}

The BPM system has total 108 output signals. The distribution of BPM in storage ring and the structure of a single BPM can be see Fig.1. The storage ring is divided into four quadrants. Each quadrant has 5-8 BPMs, which provide up to 32 output signals. The multiplexer system has three levels. Each quadrant has a sub-multiplexer (100c1423 relay switch) system. Each sub-multiplexer system has 5-8 multiplexer, which acquires 4 pick-up 
signals (total 16 for 4 quadrants). These 16 signals are then delivered to the central control room to a higher lever 4-multiplexer system via a long cable about 50 meters. Each time, the higher level system acquires 4 signals. Finally, one muliplexer processes these 4 signals. The rf cavity of the storage ring has frequency of 204 $\mathrm{MHz}$, therefore a narrow bandwidth filter, which has a centre frequency of $204 \mathrm{MHz}$ with only $10 \mathrm{MHz}$ bandwidth, is used as first stage. This $204 \mathrm{MHz}$ single frequency signal then is sent to a linear amplifier. The signal again goes through a low-pass filter and an A to D converter. The DC voltage reading is finally collected by computer. The $10 \mathrm{MHz}$ narrow band filter has greatly increased the accuracy and reliability of the system. The block diagram of the signal electronics circuit is shown in Figure 2.

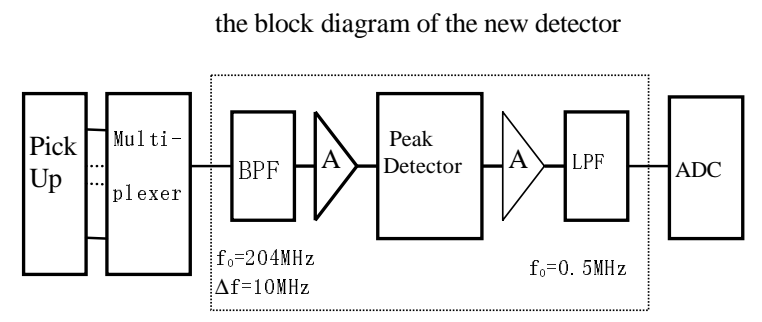

Figure2: The signal processing electronics

\subsection{Specification of the electronics}

In order to characterise the electronics system, we use a HP-8648A digit signal generator and a HP-3456 digit voltmeter for the measurement. The results indicate that $\mathrm{S} / \mathrm{N}$ of new electronics circuit is as larger than $50 \mathrm{db}$. The system has dynamic range of $33 \mathrm{db}$. The non-linearity is less than $1 \%$ in the dynamic range. The input/output and the stability of the gain of the electronics are plotted in Figure3 (a) and (b).
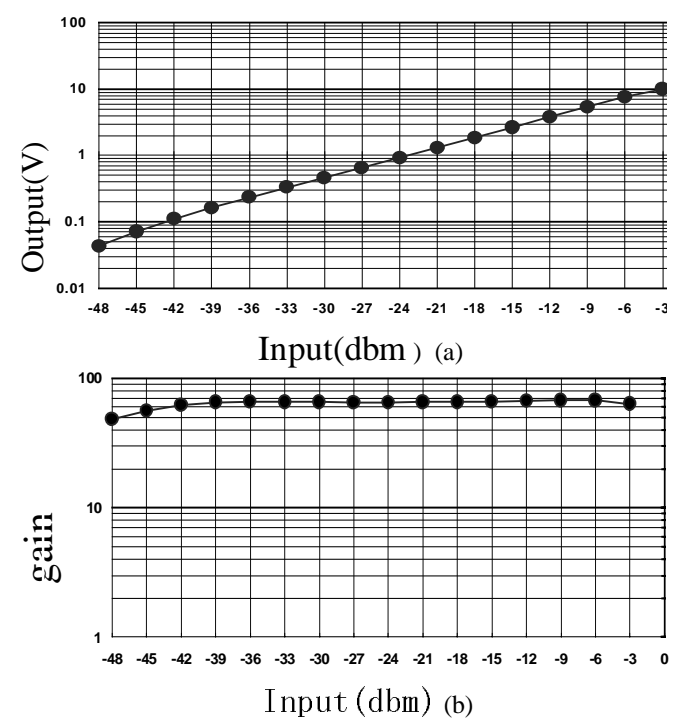

Figure 3: Character curve of $\mathrm{I} / \mathrm{O}$ and Stability of gain

\section{ANALYSIS OF THE MEASURING RESULT TO THE BPM SYSTEM}

\subsection{Experiment Result}

We have done a lot of testing regarding the repeatability and reliability of the BPM system. The test results show that the BPM readings are reproducible in $30 \mu \mathrm{m}$ and the biggest deviation of beam position ( $x$ and $y$ ) was below $50 \mu \mathrm{m}$ with beam current reducing from $150 \mathrm{~mA}$ to $68 \mathrm{~mA}$ during 10 hours. This also shows that it has little dependent on beam intensity see Figure 3 .

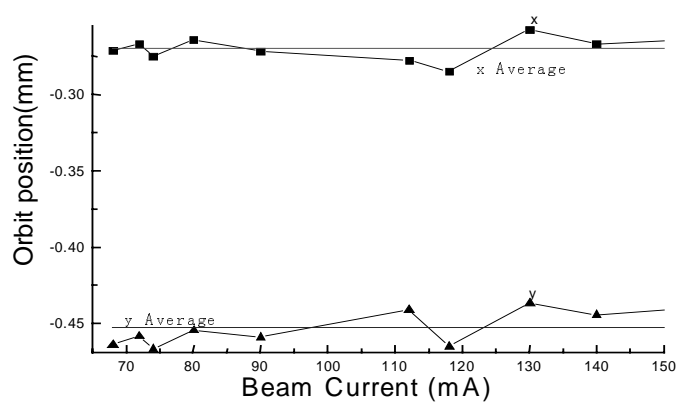

Figure 4: Deflect of the orbit with current

The system resolution can be expressed with the following formula:

$$
R=3 N_{\text {RYS }} \times 1 / S
$$

Where, $N_{R M S}$ is mean square root noise level, $S$ is the system sensitivity. The measured value is close to the one calculated based on this formula, which is about $10 \mu \mathrm{m}$, the resolution of the BPM system.

\subsection{Closed orbit measurement}

On the other hand, we use the induced signals on the button electrodes to determine the reliability of the system. The peak voltage detected by BPM button electrode can be calculated by the following formula [4]:

$V_{1,2,3,4}=\frac{d^{2}}{8 a} \cdot \frac{R \bar{I} c}{\sqrt{2 \pi} \sigma^{2} f_{0} \sqrt{e}} \cdot F\left(\delta, \theta_{1,2,3,4}\right)$

Where $F\left(\delta, \theta_{1,2,3,4}\right)$ is a beam position function, $a$ is BPM geometric radius and $d$ is BPM pickup radius.

If the button electrodes are working properly, $\left(V_{1}+V_{3}\right)$ and $\left(V_{2}+V_{4}\right)$ should be roughly equal for beam position change at $\mathrm{x}$ direction, the similar with $\mathrm{y}$ direction.

Therefore, $\left(V_{1}+V_{3}\right)-\left(V_{2}+V_{4}\right)$ should be close to zero. Since all the BPM sees the same beam current, the deviation of the sum signal of each BPM can be used to 
indicate the system reliability. The following are the described formula:

$$
\begin{aligned}
S & =\frac{\sum_{j=1}^{4} V_{j}}{V_{A}} \approx 1, \quad D=\frac{\left(V_{1}+V_{3}\right)-\left(V_{2}+V_{4}\right)}{V_{A}} \approx 0 \\
V_{A} & =\left(\sum_{j=1}^{4} V_{j}\right)_{\text {average for all BPM }}
\end{aligned}
$$

In our case, the measurements showed that $\mathrm{S}$ is inside $1 \pm 0.2$ and Deviation is no bigger than 0.01 for most of BPMs. Beam orbit measurements have been done with orbit change induced by the use of the magnetic correctors[5]. The measurements supported the predicted orbit changes based on the machine physics calculation, see Figure 5, Figure 6.

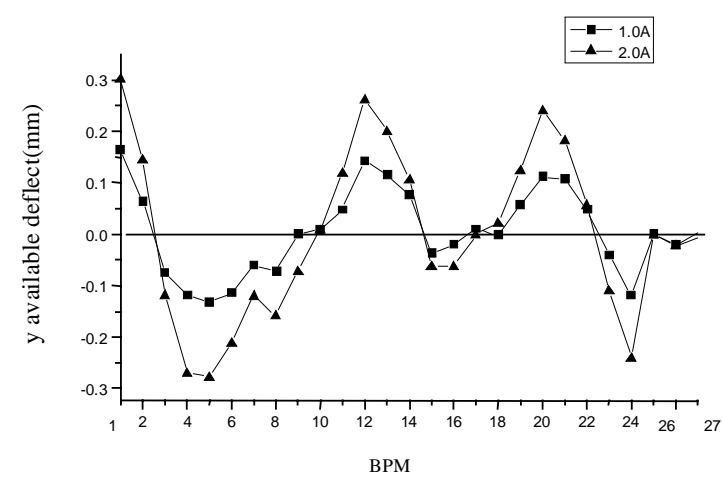

Figure 5: Relation curve of position deflect and Correct current

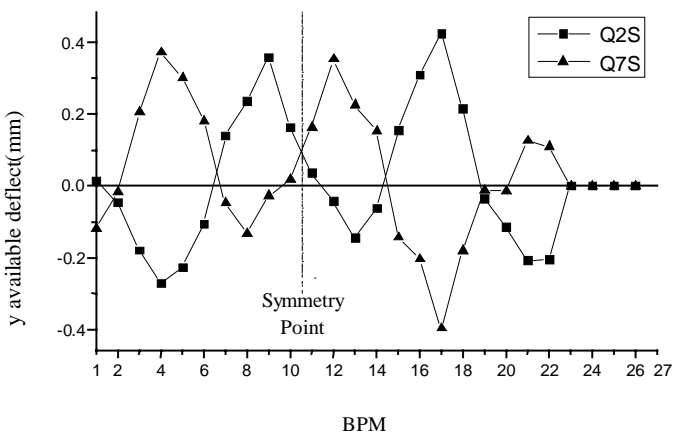

Figure 6: The analysis for system symmetry (Q2S and Q7S been separately charged)

The subsystem of the BPM for each quadrant also showed similar orbit shifts with phase difference between the quadrants when the corresponding magnetic correctors in each quadrant were in operation. The detail is shown in Figure 7.

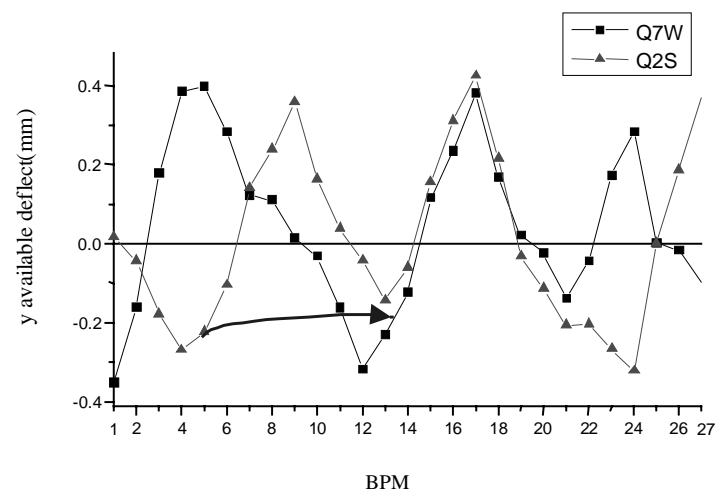

Figure 7: the analysis for system period (Q2Sand Q7W been separately charged)

\section{CONCLUSION}

The beam position monitoring system of the NSRL storage ring basically satisfies the requirements of machine operation and studies of storage ring. The shortterm reproducibility of measurement is better than $10 \mu \mathrm{m}$. The measurement time for scanning all 27 BPMs is about 15 seconds. Absolute beam position with respect to the magnetic centre of the adjacent quadrupole magnet will be determined directly with the beam in the near further.

\section{ACKNOWLEDGEMENTS}

We would like to thank Prof. C.Y. Yao of APS, Mr. Y.G. Zhou and many colleagues of NSRL, who made contributions to this work. The first author especially thanks Dr. Takao IEIRI of KEK and Prof. L. Ma of BEPC for their fruitful discussion.

\section{REFERENCE}

[1]H.N. Xu, closed-orbit correct, Paper of Doctor of NSRL, 1998. [2] J.Y.Li, Z.P.Liu, and J.H.Wang, High power Laser and Partice Beams, Vol.10 No.2(1998), P291.

[3]Y. Yin, J. S. Fan, The proceeding of the International Conference on Synchrotron Radiation Application. (May 9-12, 1989), P270.

[4] Ma Li, BPM measurement, The Symposium of Science and Technology of BEPC.

[5] M. Sands, The Physics of Electron Storage Ring, An introduction, SLAC-121 (1970). 